\title{
Quantitative Pathology of Inhalational Anthrax I: Quantitative Microscopic Findings
}

Lev M. Grinberg, M.D., Faina A. Abramova, M.D., Olga V. Yampolskaya, M.D., David H. Walker, M.D., Jerome H. Smith, M.D.

Department of Pathology, Tuberculosis and Pulmonary Diseases Unit (LMG, FAA), Hospital 40, Ekaterinburg, Russia; Department of Infectious Diseases, Central Postgraduate Institute, Botkin Hospital (OVY), Moscow, Russia; and Department of Pathology, University of Texas Medical Branch (DHW, JHS), Galveston, Texas

Forty-one cases of documented inhalational anthrax from the Sverdlovsk epidemic of 1979 traced to release of aerosols of Bacillus anthracis at a secret biologic-agent production facility were evaluated by semiquantitative histopathologic analysis of tissue concentrations of organisms, inflammation, hemorrhage, and other lesions in the mediastinum, mediastinal lymph nodes, bronchi, lungs, heart, spleen, liver, intestines, kidneys, adrenal glands, and central nervous system. These data were correlated with clinical, epidemiologic, and demographic data.

The patients' courses, with a variable incubation period and short nonspecific course ( 4 days before hospitalization) with rapid demise (1 day of hospitalization before death), correlated with systemic bacterial infection and lesions. Bacillus anthracis were identified in all cases in which there was no antibiotic treatment or there was treatment for fewer than 21 hours. The lesions that were the most severe and apparently of longest duration were in the mediastinal lymph nodes and mediastinum. There and elsewhere, peripheral transudate surrounded fibrin-rich edema; necrosis of arteries and veins was the most likely source of large hemorrhages displacing tissue or infiltrating tissue, respectively; and apoptosis of lymphocytes was observed. Respiratory function was compromised by mediastinal expansion, large pleural effusions, and hematogenous and retrograde lymphatic vessel spread of $B$. anthracis to the lung with consequent pneumonia. The central nervous system and intestines manifested similar hematogenous spread, vas-

Copyright (C) 2001 by The United States and Canadian Academy of Pathology, Inc.

VOL. 14, NO. 5, P. 482, 2001 Printed in the U.S.A.

Date of acceptance: January 22, 2001.

Address reprint requests to: David H. Walker, M.D., WHO Collaborating Center for Tropical Diseases, University of Texas Medical Branch, 301 University Boulevard, Galveston, TX 77555-0609; e-mail: dwalker@utmb.edu; fax: 409-772-2500. culitis, hemorrhages, and edema. These pathologic findings are consistent with previous experimental studies showing transport of inhaled spores to mediastinal lymph nodes, where germination and growth lead to local lesions and systemic spread, with resulting edema and cell death, owing to the effects of edema toxin and lethal toxin. The identification of the vascular lesions as a basis for the prominent hemorrhages is a novel observation for human inhalational anthrax.

KEY WORDS: Bacillus anthracis, Edema, Hemorrhage, Inhalational anthrax, Mediastinum, Respiratory failure, Vasculitis.

Mod Pathol 2001;14(5):482-495

An epidemic of inhalational anthrax occurred in Sverdlovsk (now Ekaterinburg), Russia in April and May of 1979 (1-3). This outbreak was initially reported to be intestinal anthrax $(4,5)$, but lay press investigation raised questions $(6-8)$ that culminated in an international study, which established that the outbreak was caused by at least four strains of Bacillus anthracis (3), documented that the pathologic findings were those of inhalational anthrax (1), and uncovered by an epidemiological investigation a high likelihood that the origin was a military base, Compound 19, in southern Sverdlovsk (2). Forty-two deceased patients were autopsied during that epidemic (1). This is the largest series of human inhalational anthrax that has undergone postmortem analysis; the next largest series was a cluster of three deaths $(9,10)$, and other individual case autopsies have also been reported (11-15).

The pathogenesis of inhalational anthrax has been studied in experimental animals (16-21). Inhaled spores are phagocytosed by alveolar macrophages and carried to regional lymph nodes in the mediastinum within 6-18 hours in guinea pigs (18), rhesus monkeys (19), and chimpanzees (20). Ger- 
mination of spores and multiplication of bacilli occur in these lymph nodes, and vegetative bacteria spread via lymphohematogenous route to disseminated loci (21). A similar pattern was found in a human series $(9,21)$. More recently, $B$. anthracis has been shown to elaborate three proteins that comprise two toxins: protective antigen (PA), which binds to cell surfaces and mediates internalization of the proteins; edema factor (EF), and lethal factor (LF; 22). EF acts as an $\mathrm{Ca}^{+2}$ - and calmodulindependent adenylate cyclase which induces edema (22). LF is a metalloprotease that cleaves the amino terminus of MAP kinase kinases 1 and 2 and thus inhibits the intracellular MAPK signal transduction pathway (23), which may favor apoptosis. A sublethal concentration of LF also causes macrophages to produce TNF- $\alpha$ and IL-1 (24), which might induce pathologic effects.

Previous reports of this series focused upon the gross (macroscopic) findings $(1,24)$; the microscopic findings reported here have been published in abbreviated form in the Russian literature (2527 ) and in abstracts of presentations (28-30). The present report summarizes the quantitated microscopic findings in human inhalational anthrax and correlates these with results of experimental studies $(16-21)$ and previous reports of human inhalational $(9-15)$ and intestinal anthrax $(31,32)$.

\section{MATERIALS AND METHODS}

Gross and microscopic specimens, as well as some clinical information, were available for 42 patients who underwent postmortem examination during the epidemic and were thought clinically to have inhalational anthrax, as reported earlier (1). In one long survivor (Case 24), clinical evidence of $B$. anthracis infection was not available, and there was no objective evidence of $B$. anthracis infection at autopsy after a full 8 days of antibiotic therapy and clinical recovery from the apparent anthrax illness (she presumably died of complications from the anthrax). In view of the tenuous nature of the evidence that her illness was indeed anthrax, we have excluded this patient's case from this series.

Each of the following epidemiological variables was extracted from available records and tabulated: name, age, sex, duration of known antibiotic therapy (in hours), estimated date of exposure, date of onset of illness, date of admission to Hospital 40, date of death, incubation period (in days), duration of illness (in days), duration of hospitalization (in days), overall duration of infection from exposure to death (in days), postmortem interval (in hours), culture results for $B$. anthracis, and histological detection of large Gram-positive bacilli morphologically consistent with $B$. anthracis.
After initial examination of microscopic slides cut from preserved paraffin blocks and formalin fixed tissues, the slides were reviewed by two of the authors (JHS and LG) on a double-headed microscope, and the microscopic features itemized in Tables 4-10 were semiquantified (scale of 1-4; see below) and tabulated.

\section{Definition of Terms}

Hemorrhage was differentiated into highpressure hemorrhage and low-pressure hemorrhage based on whether aggregates of "pure" red cells displaced and compressed adjacent tissue (high-pressure hemorrhage) or whether red blood cells diffusely permeated parenchyma without distorting its architecture (low-pressure hemorrhage). The Russian literature on pathology of anthrax (which is more extensive than that of Western medical literature) differentiates macroscopically between carbuncles and diffuse hemorrhage in the gastrointestinal tract; carbuncles are discreet, often circular areas of mucosa elevated by high-pressure hemorrhage. The term carbuncle has a quite different usage in the modern English medical literature, but its meaning did include hemorrhage in the archaic English medical literature, We prefer the term submucosal hemorrhage to the translated Russian term carbuncle for these lesions. Diffuse gastrointestinal hemorrhage is flat, but hemorrhagically discolored, mucosa and correlates with lowpressure hemorrhage in the submucosa, as opposed to superficial hemorrhagic necrosis, which is hemorrhage into the lamina propria, resulting from reperfusion.

The tissue concentrations of $B$. anthracis, in other words, Gram-positive bacilli/25× objective field, were semiquantified as in Table 1 . The tissue concentration of inflammatory cells was semiquantified as described in Table 2. Other parameters, such as congestion and transudate were quantified as defined in Table 3.

Additionally, selected blocks were stained with periodic acid-Schiff/Luxol fast blue, Mallory's trichrome, phosphotungstic acid-hematoxylin, periodic acid-methenamine silver, Perl's (iron), Brown-Brenn, Brown-Hopps, Dieterle, Gridley's methenamine silver, hematoxylin-azure II-eosin, Giemsa, Ziehl-Neelsen, Verhoef-Van Giesen, Movat

\begin{tabular}{cll} 
TABLE 1. Criteria for Quantification of Tissue & \\
Concentrations of Bacillus anthracis & \\
\hline 0 & Bacillus anthracis $/ 25 \times$ objective field & $=0$ \\
$<1$ & Bacillus anthracis $/ 25 \times$ objective field & $=1$ \\
$1-10$ & Bacillus anthracis $/ 25 \times$ objective field & $=2$ \\
$11-100$ & Bacillus anthracis $/ 25 \times$ objective field & $=3$ \\
$101-500$ & Bacillus anthracis $/ 25 \times$ objective field & $=4$ \\
501 or more & Bacillus anthracis $/ 25 \times$ objective field & $=5$ \\
\hline
\end{tabular}


TABLE 2. Criteria for Quantification of Tissue

Concentrations of Inflammatory Cells

0 cells $/ 25 \times$ objective field

$>0-10$ cells $/ 25 \times$ objective field

11-25 cells $/ 25 \times$ objective field

$26-100$ cells $/ 25 \times$ objective field

$>100$ cells $/ 25 \times$ objective field

pentachrome, Rebiger's and Mekhin stains for bacterial capsules, and Schaeffer-Fulton modification of Wirtz spore stains.

Immunohistochemical assessment of hepatitis B viral core and surface antigens, fibrinogen, IgG, IgM, C3 complement, B. anthracis antigens, CD45 (LCA), S-100, L26, CD45 RO, and KP1 was performed on selected blocks, using positive and negative controls from University of Texas Medical Branch archival tissues.

For the immunocytochemical assessment of putative anthrax bacilli in mediastinal lymph nodes and cerebral meninges, rabbit antibody (primary antibody) against $B$. anthracis (Vollum strain) spores (provided by Dr. Martin Hugh-Jones, Louisiana State University, Baton Rouge, LA) was incubated with deparaffinized, rehydrated, proteasedigested $7-\mu \mathrm{m}$ sections; after washing, horseradish peroxidase-conjugated goat anti-rabbit antibody (secondary antibody) was applied, and after incubation, unbound antibody was rinsed off with APK wash buffer using standard protocols for the Ventana 320 immunohistologic automated stainer. The positive control was a 3-day-old injection site of an anthrax immunization in the skin and subcutaneous tissue of the arm in one of the cases (polymerase chain reaction testing [PCR] [3] on this lesion showed a single vaccine strain of $B$. anthracis); negative control was the section of colon and luminal feces containing a variety of bacteria from another patient in this series.

All data were entered into a Microsoft Excel 5.0 for Windows spreadsheet program on one of several IBM-clone desktop computers and analyzed with the standard statistical packages available in the Data Analysis section of the program's Tools menu. Quantitative data presented in the text and tables are the mean \pm SEM unless otherwise specified.

TABLE 3. Criteria for Quantification of Other Parameters

\begin{tabular}{|c|c|c|}
\hline 0 & $=$ Absent & \\
\hline $1 / 2$ & $=$ Minimal & $=$ Present, but barely evident \\
\hline 1 & $=$ Mild & $\begin{aligned}= & \text { Clearly present, but probably } \\
& \text { pathophysiologically insignificant }\end{aligned}$ \\
\hline 2 & $=$ Moderate & $=$ Present and significant \\
\hline 3 & $=$ Marked & $\begin{aligned}= & \text { Prominent, but without destruction of the organ } \\
& \text { architecture }\end{aligned}$ \\
\hline 4 & $=$ Severe & $=$ Prominent with organ architecture destruction \\
\hline
\end{tabular}

\section{RESULTS}

Bacillus anthracis organisms were observed in most organs examined microscopically in cases treated with antibiotics for fewer than 21 hours or not treated with antibiotics at all. The bacilli (Fig. 1) were large ( 1 to $2 \times 8$ to $12 \mu \mathrm{m}$ ), often curved, in sausage-like chains, and usually Gram-positive (on Brown-Brenn or Brown-Hopps stains), without spores (Schaeffer-Fulton modification of Wirtz spore stains and electron microscopy) or capsules detected by light microscopy (Rebiger's and Mekhin stains). The bacilli stained well with Dieterle's stain, weakly with Gridley's methenamine silver, and blue with hematoxylin-azure II-eosin, Giemsa and Ziehl-Neelsen stains. In some specimens, nearly all of the bacilli appeared Gram-negative or partially Gram-negative. Bacilli from cases in which bacilli stained Gram-negative or Gram-variable, as well as cases in which bacilli were typical Grampositive bacilli, stained immunohistochemically with rabbit antibody to $B$. anthracis (Vollum strain) spores. Bacilli pervaded soft tissues with predilection for lymphangioles. In the lungs of some cases, aggregates of bacilli appeared to obstruct capillaries and venules. In the brain, bacilli often surrounded blood vessels, imparting a felt-work zone interposed between the vascular adventitia and the adjacent neuropil. Bacilli were often observed at the highest concentration in the middle of lesions progressively diminishing in concentration toward the periphery, but high-pressure hemorrhage disrupted such central concentrations.

Epidemiological, clinical, and general pathologic data and microscopic findings for all cases are shown in Tables 4-10. Many of the values exhibited large variances, which appeared to be due to differences between those patients with known antibiotic treatment and those without.

\section{Epidemiological Data}

The age was available in $98 \%$ of patients; the mean age was 46 years old (range, 25 to 71 years). There were 33 men and 8 women. Twenty-two of 41 (54\%) of patients were known to have received antibiotic therapy. The incubation period could be determined in 30 of 41 (73\%) of patients; the mean incubation period was 16 days. Duration of clinical illness and duration of hospitalization were known in $73 \%$ and $90.2 \%$ of cases, respectively; the average duration of clinical illness and duration of hospitalization were 3.85 days and 1 day, respectively. The mean overall duration of the infection from putative exposure to death was known in all cases and was 19.5 days, but there was a wide range from 6 days to 69 days. The mean postmortem interval (which bears upon the validity of the microscopic 


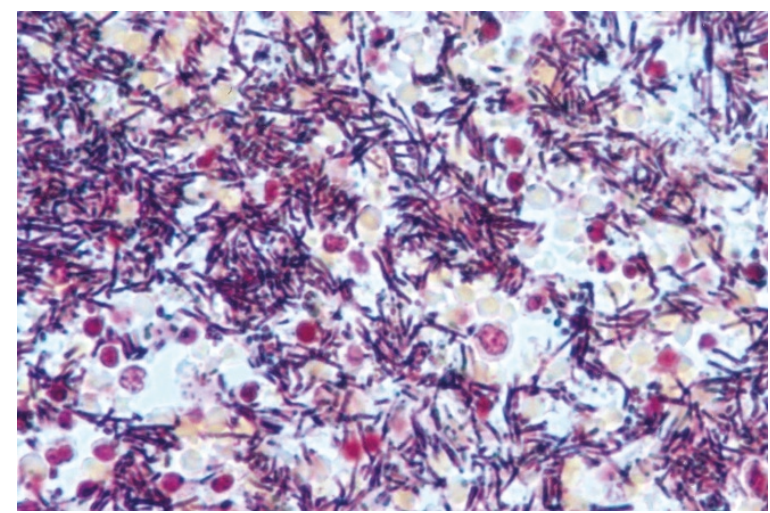

FIGURE 1. Photomicrograph of Bacillus anthracis in tissue of short survivor (death occured 2 days after onset of illness). Brown-Hopps stain, $500 \times$

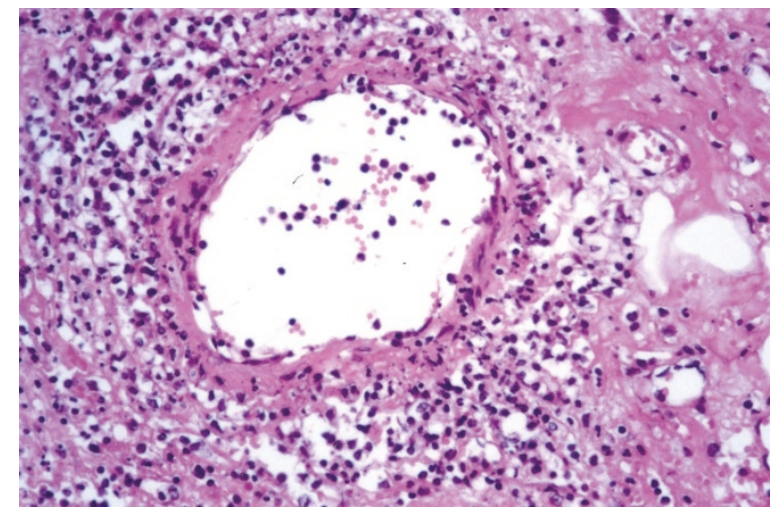

FIGURE 2. Photomicrograph of mediastinal arterial branch in a short-surviving patient (death occurred 3 days after onset of illness) with acute vasculitis characterized by fibrinoid necrosis and mixed, predominantly neutrophil infiltrates. Hematoxylin and eosin staining; $100 \times$

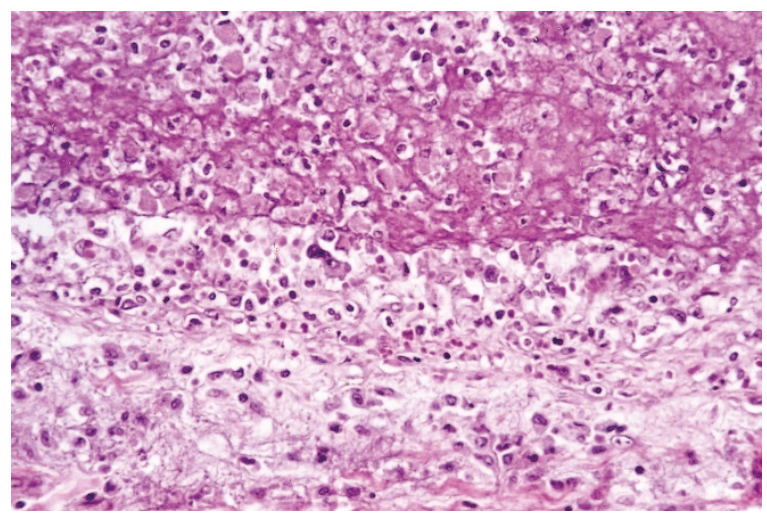

FIGURE 3. Photomicrograph of arterial branch in a longer surviving patient (death occurred 8 days after onset of illness) with vasculitis characterized by mononuclear infiltrates of intima, media and adventitia; thrombosis in lumen is noted (upper portion of figure) Hematoxylin and eosin staining; $50 \times$.

data) was known in $88 \%$ and was approximately 19 hours. See Table 6.

\section{General Pathologic Data}

Vasculitis (Table 4 and Figs. 2-4) was very evident in some specimens and some cases; it was probably

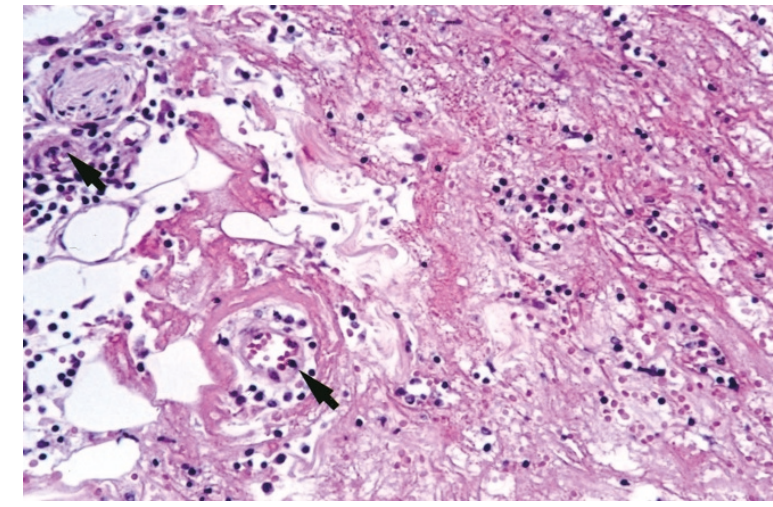

FIGURE 4. Photomicrograph of capillaritis (arrows) and fibrin-rich edema in interstitium of mediastinum in patient with short survival (death occurred 3 days after onset of illness). Hematoxylin and eosin staining; $300 \times$

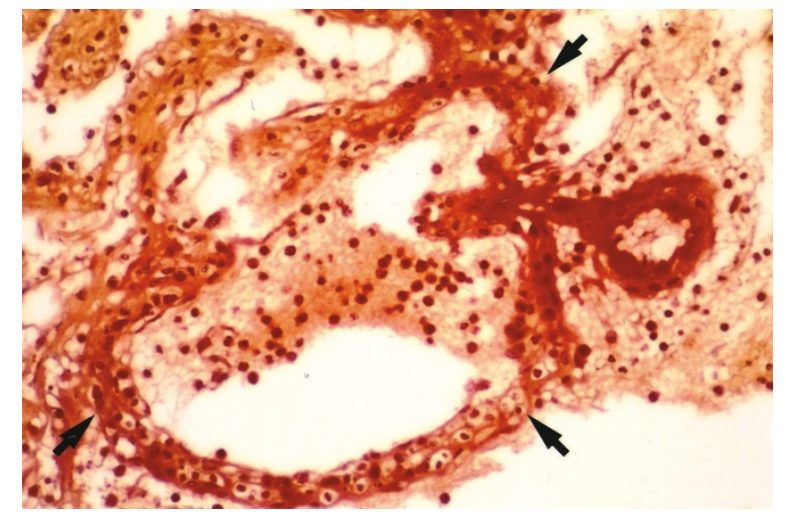

FIGURE 5. Photomicrograph of microaneurysm (arrows) in a small artery with acute vasculitis in a short-surviving patient (death occurred 3 days after onset of illness). Brown-Hopps staining; $300 \times$.

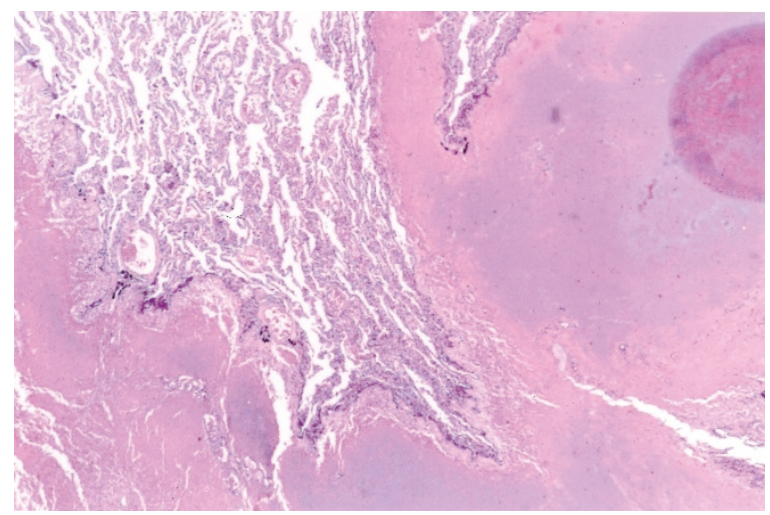

FIGURE 6. Photomicrograph of high-pressure hemorrhage compressing adjacent lung tissue and distorting its architecture. Hematoxylin and eosin staining; $20 \times$.

present in all cases, but its focality predisposed to sampling error. Both arteries and veins as well as intervening smaller vessels were involved. Larger vessels in patients dying early in the course of the disease presented vasculitis, which was characterized by loss of nuclei in the smooth muscle cells of the media, necrosis associated with fibrin (Biebrich scarlet staining in Mallory's trichrome; dark purple 
TABLE 4. Quantitative Microscopic Findings in Inhalational Anthrax: Epidemiologic, Clinical and General Pathologic Observations

\begin{tabular}{lcc}
\hline & Mean \pm SD & \% with Lesion \\
\hline Age (years) & $46.13 \pm 1.99$ & \\
Gender (males $=1$; females = 2) & $1.195 \pm 0.062$ & \\
Antibiotic therapy (h) & $16.61 \pm 5.85$ & \\
Incubation period (d) & $16.07 \pm 1.80$ & \\
Duration of clinical illness (d) & $3.85 \pm 0.41$ & \\
Duration of hospitalization (d) & $0.96 \pm 0.27$ & \\
Overall interval from exposure to death (d) & $19.54 \pm 1.93$ & \\
Postmortem interval (h) & $18.82 \pm 2.94$ & 73 \\
Volume of bilateral pleural fluid (mL) & $1776 \pm 177$ & 78 \\
Vasculitis and capillaritis (0 to 5 scale) & $3.448 \pm 0.49$ & \\
Vasculitis only (0 to 5 scale) & $2.45 \pm 0.41$ & \\
High pressure hemorrhage (0 to 5 scale) & $3.46 \pm 0.55$ & \\
Hemorrhagic diathesis & $0.73 \pm 0.07$ & \\
Bacillus anthracis burden (sum of all & $11.68 \pm 1.81$ & \\
$\quad$ organs) & & \\
\hline
\end{tabular}

by phosphotungstic acid-hematoxylin; red with Movat's pentachrome; positive for fibrinogen by immunohistochemical method), and variable neutrophil infiltrates; in patients surviving longer, mononuclear cells predominated in the intima, adventitia, and perivascular space. At one site, a small artery with vasculitis had a saccular aneurysm (Fig. 5), and in another vessel, a rupture of a similar "microaneurysm" was noted. The latter lesions may be the origin of the high-pressure hemorrhage noted, but these hemorrhages were much larger and more frequent than observed foci of vasculitis or aneurysms (Table 4). High-pressure hemorrhage (Fig. 6) was seen in 32 of 41 cases; low-pressure hemorrhages were seen in all cases. Hemorrhagic diathesis was characterized by numerous petechiae, and ecchymoses in skin and serosal surfaces seen macroscopically (both clinically and at autopsy).

\section{Quantitative Microscopic Findings by Organ}

\section{Heart}

Minimal concentrations of Bacillus anthracis were identified in cardiac sections of 9 of 25 patients (Table 5), and there were no specific cardiac microscopic findings, although occasional cases showed individual myocyte hypereosinophilia and rare focal-contraction band necroses, attributed to agonal hypotension and hypoxemia.

\section{Mediastinal Lymph Nodes}

See Table 5. These contained identified B. anthracis in 21 of 35 cases (Fig. 7). There was marked lymphocytolysis (Fig. 8), which was morphologically apoptotic, in both follicular and paracortical areas. There was prominent phagocytosis of lymphocytes by macrophages (emperipolesis) in 13 of 29 cases and/or edema (12 of 25 patients), but the average severity of these lesions was minimal. Mild inflammation was present and consisted of neutrophils, macrophages, and fibrin-rich edema, which is the microscopic counterpart of grossly observed "gelatinous" edema (Fig. 9a). Fibrin-rich edema (Fig. 9b) appeared as strands of fibrin (Biebrich scarlet staining by Mallory's trichrome; phosphotungstic acid-hematoxylin positive; red with Movat's pentachrome; positive for fibrinogen in immunohistochemical method) coursing through eosinophilic homogeneous "fluid." The density and eosinophilia of the continuous phase progressively diminished from the center to periphery of lesions. There was low-pressure hemorrhage into mediastinal lymph nodes in a higher proportion of patients and to a more severe degree than high-pressure hemorrhage. Hyperplasia of what appeared to be large lymphocytes was observed mostly in patients who succumbed later in the disease's course. These round to ovoid cells were up to $20 \mu \mathrm{m}$ in diameter, with basophilic cytoplasm and hyperchromatic nu-

TABLE 5. Quantitative Microscopic Findings in Heart, Mediastinal Lymph Nodes, Mediastinum, and Peribronchial Soft Tissue in Inhalational Anthrax

\begin{tabular}{|c|c|c|}
\hline & Severity & $\%$ with Feature \\
\hline \multicolumn{3}{|l|}{ Heart } \\
\hline Bacillus anthracis burden & $0.56 \pm 0.20$ & 36 \\
\hline \multicolumn{3}{|l|}{ Mediastinal lymph nodes } \\
\hline B. anthracis burden & $1.493 \pm 0.274$ & 60 \\
\hline \multicolumn{3}{|l|}{ Lymphocytolysis } \\
\hline Follicular & $2.936 \pm 0.188$ & 94 \\
\hline Paracortical & $3.186 \pm 0.224$ & 89 \\
\hline Low-pressure hemorrhage & $2.671 \pm 0.180$ & 94 \\
\hline \multicolumn{3}{|l|}{ hemorrhage } \\
\hline Emperiopolesis & $0.444 \pm 0.099$ & 45 \\
\hline Hyperplasia & & 54 \\
\hline Sinusoidal & $0.118 \pm 0.067$ & \\
\hline Follicular & $0.059 \pm 0.059$ & \\
\hline Paracortical & $0.059 \pm 0.059$ & \\
\hline Atrophy & $0.147 \pm 0.058$ & \\
\hline Edema & $0.485 \pm 0.134$ & 48 \\
\hline \multicolumn{3}{|l|}{ Inflammation } \\
\hline Neutrophils & $1.570 \pm 0.202$ & 82 \\
\hline Mononuclear cells & $0.770 \pm 0.160$ & 60 \\
\hline Fibrin-rich edema & $1.100 \pm 0.166$ & 69 \\
\hline $\begin{array}{l}\text { Large lymphocyte } \\
\text { hyperplasia }\end{array}$ & \multicolumn{2}{|c|}{ hyperplasia } \\
\hline Vasculitis & $0.600 \pm 0.159$ & 36 \\
\hline Fibrin & $1.962 \pm 0.237$ & 92 \\
\hline Neutrophils & $1.077 \pm 0.193$ & 92 \\
\hline Mononuclear cells & $1.115 \pm 0.378$ & 69 \\
\hline \multicolumn{3}{|l|}{$\begin{array}{l}\text { Mediastinum and } \\
\text { peribronchial soft tissue }\end{array}$} \\
\hline B. anthracis burden & $1.375 \pm 0.269$ & 54 \\
\hline Fibrin-rich edema & $2.146 \pm 0.179$ & 92 \\
\hline Low-pressure hemorrhage & $2.274 \pm 0.170$ & 95 \\
\hline $\begin{array}{l}\text { High-pressure } \\
\text { hemorrhage }\end{array}$ & $1.368 \pm 0.244$ & 59 \\
\hline Neutrophils & $1.061 \pm 0.153$ & 81 \\
\hline Mononuclear cells & $0.702 \pm 0.112$ & 76 \\
\hline Lymphangitis & $1.472 \pm 0.236$ & 65 \\
\hline Vasculitis & $0.681 \pm 0.168$ & 46 \\
\hline Bacterial obstruction & $0.259 \pm 0.165$ & 11 \\
\hline Fibrin & $2.000 \pm 0.272$ & 87 \\
\hline Neutrophils & $0.983 \pm 0.185$ & 87 \\
\hline Mononuclear cells & $0.960 \pm 0.310$ & 67 \\
\hline
\end{tabular}




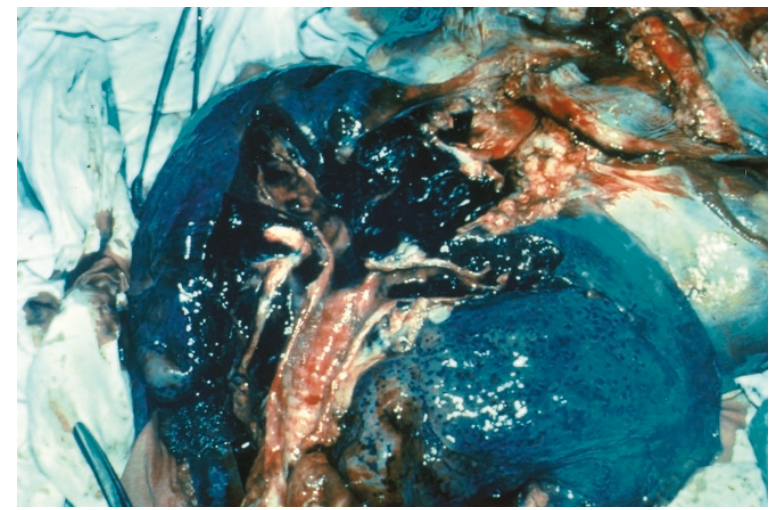

FIGURE 7. Gross photograph of hemorrhagic mediastinal lymphadenitis and mediastinitis and lungs of fatal inhalational anthrax patient.

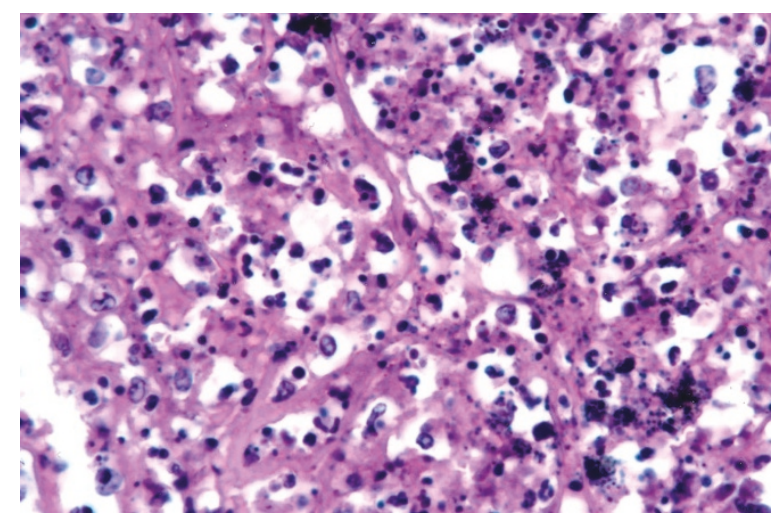

FIGURE 8. Photomicrograph of lymphocytolysis (and low-pressure hemorrhage) in mediastinal lymph node of patient succumbing to fatal inhalational anthrax. Hematoxylin and eosin staining; $200 \times$.

clei, and stained immunocytochemically with CD45 (LCA) and S-100. Vasculitis within mediastinal lymph nodes consisted of a minimal degree of fibrinoid necrosis, neutrophils, and macrophages. In all cases, the lesions in the mediastinal lymph nodes and mediastinal soft tissues appeared most extensive and/or older than in other sites in the body, but this was not quantifiable.

\section{Mediastinum and Peribronchial Soft Tissues}

See Table 5. These contained identifiable B. anthracis in 20 of 37 cases. Bacilli were identified in lymphangioles in 24 of 37 cases with a decreasing gradient extending from heavily burdened lymph nodes to lesser quantities of organisms in the pulmonary hilum and lungs (retrograde extension) and downstream toward the thoracic duct. There was moderate fibrin-rich edema and low-pressure hemorrhage, mild and less frequent high-pressure hemorrhage, and mild neutrophilic and mononuclear infiltrates and mild-to-moderate lymphangitis. There was only a minimal degree of vasculitis characterized by fibrinoid necrosis, neutrophils, and mononuclear cells (predominantly lymphocytes and plasma cells). Vasculitis in 3 of 28 short survivors produced obstruction of blood vessels by aggregates of $B$. anthracis with preobstructive dilatation and postobstructive collapse.

\section{Bronchi}

See Table 6 . These contained B. anthracis in 10 of 28 cases. Ulceration, pseudomembrane formation, and squamous metaplasia were observed infrequently with mild acute bronchitis (neutrophil infiltrates, congestion, and edema) and mild chronic bronchitis (bronchial basement membrane thickening, mononuclear infiltrates, increased goblet cells, and bronchial gland hyperplasia occurring in most of the cases). Squamous metaplastia and chronic bronchitis were presumably underlying conditions. There was mild fibrin-rich edema and low-pressure hemorrhage in bronchi and minimal high-pressure hemorrhage. Bronchial vasculitis noted infrequently was characterized by fibrinoid necrosis, neutrophils, and mononuclear cells (predominantly lymphocytes and plasma cells).
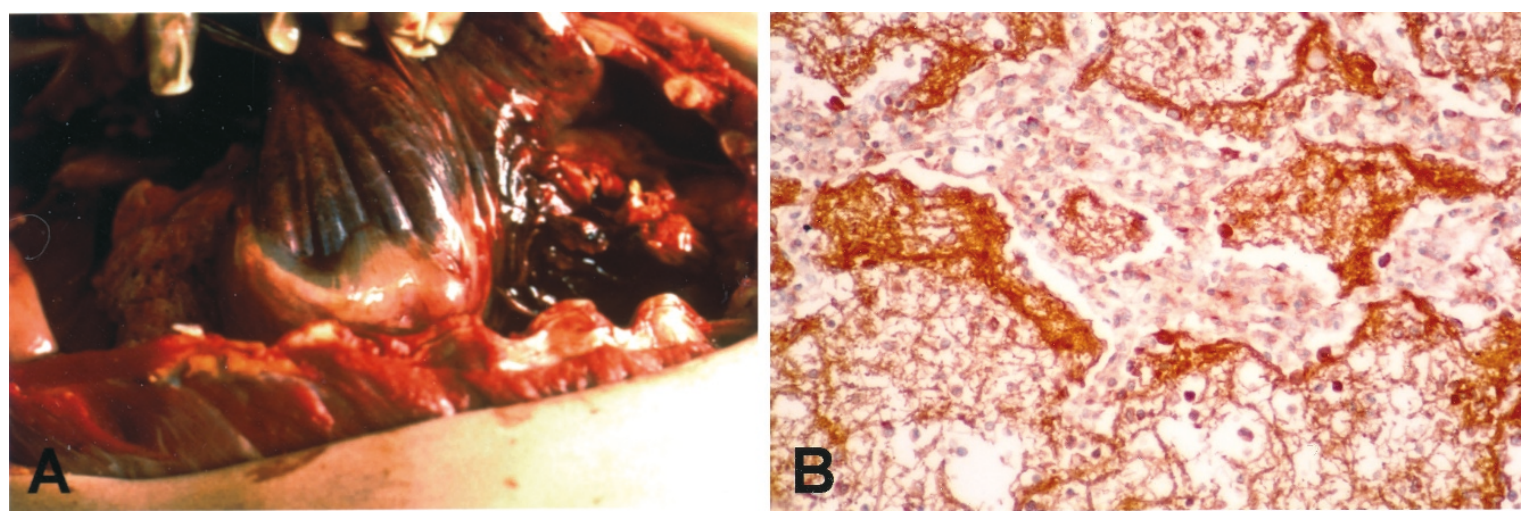

FIGURE 9. Gelatinous or fibrin-rich edema. A, Macroscopic appearance of gelatinous edema spreading from mediastinum along dorsal costal parietal pleura. B, Photomicrograph of fibrin-rich edema in mediastinum of patient with fatal inhalational anthrax. Strands of positively staining material comprise a network within the edema-expanded interstitium. Immunohistochemical stain with antifibrinogen antibodies; $100 \times$. 


\section{Lungs}

See Table 7. These contained B. anthracis in 17 of 35 cases. Over half of the bacilli were in alveolar septal capillaries and venules; less than a fifth were in alveolar air spaces, and a quarter were in the extravascular interstitium of the alveolar septa. Thus, there appeared to be a gradient from blood to air space, suggesting that pulmonary involvement by vegetative bacilli was of hematogenous origin, rather than endobronchial origin. There was perihilar interstitial pneumonia in all lungs, presumably an extension of the process originating in the mediastinal lymph nodes and mediastinal soft tissues; this perihilar interstitial pneumonia extended into the air spaces in some, but not all, patients.

Independent of the perihilar process, there was acute bronchopneumonia in nearly half of the patients. Acute bronchopneumonia involved all five lobes of lung in three patients, the entire left lung in one case, left upper lobe only in two patients, left lower lobe only in 3 patients, right middle and lower lobes only in 2 patients, right upper and lower lobes only in 1 case, right lower lobe only in 2 patients, right upper lobe only in 3 cases and right middle lobe only in 1 patient. In the other 8 patients, acute bronchopneumonia was only identified microscopically, and the specific lobe involved was not identified. Microscopic characteristics of the bronchopneumonia are best described according to each of four microanatomic compartments: intra-alveolar exudate, interstitial exudate, capillaries, and lymphangioles. Alveolar spaces contained mild transudate, mild fibrin exudate, mild hemorrhage, and mild macrophage infiltration; there was minimal neutrophil infiltration, minimal lymphocyte infiltration, minimal plasma cell infiltration, and minimal nuclear debris. Alveolar interstitium was expanded by mild transudation, mild fibrin deposition, mild hemorrhage, and mild infiltration by macrophages with minimal neutrophil infiltration, minimal lymphocyte infiltration, and minimal plasma cell infiltration but negligible nuclear debris. Capillaries (and venules) were moderately

TABLE 6. Quantitative Microscopic Findings in Bronchi in Inhalational Anthrax

\begin{tabular}{lcc}
\hline & Severity & $\begin{array}{c}\% \text { with } \\
\text { Feature }\end{array}$ \\
\hline Bacillus anthracis burden & $0.917 \pm 0.239$ & 36 \\
Ulceration & $0.036 \pm 0.036$ & 4 \\
Pseudomembranous tracheobronchitis & $0.0185 \pm 0.0185$ & 4 \\
Squamous metaplasia & $0.098 \pm 0.052$ & 14 \\
Chronic bronchitis & $1.389 \pm 0.165$ & 81 \\
Acute bronchitis & $1.223 \pm 0.186$ & 81 \\
Fibrin-rich edema & $1.134 \pm 0.192$ & 64 \\
Low-pressure hemorrhage & $1.375 \pm 0.231$ & 71 \\
High-pressure hemorrhage & $0.304 \pm 0.198$ & 11 \\
Vasculitis & $0.185 \pm 0.120$ & 11 \\
$\quad$ Fibrin & $1.333 \pm 0.441$ & \\
Neutrophils & $0.833 \pm 0.583$ & \\
$\quad$ Mononuclear & $1.667 \pm 0.882$ & \\
\hline
\end{tabular}

congested. There was mild capillaritis in the majority of cases characterized by minimal fibrinoid necrosis, mild to moderate neutrophilia, and minimal-to-mild infiltration by macrophages. Pulmonary vasculitis was identified in the majority of cases but was minimal in severity, composed of infrequent foci of fibrinoid necrosis, mild neutrophil infiltration, and moderate infiltration by macrophages. Lymphangioles in the interstitium were mildly to moderately dilated in most cases, with mild acute lymphangitis and minimal hemorrhage; in a few patients, lymphatic channels were obstructed by aggregates of bacilli, and the average degree of involvement by this feature was minimal.

\section{Spleen}

See Table 8 . The spleen contained B. anthracis in 18 of 35 patients. White pulp: lymphoid follicles (B-cell areas) contained moderate to marked lymphocytolysis, consistent with apoptosis, whereas periarteriolar lymphatic sheaths (T-cell areas) showed marked lymphocytolysis. Follicles exhibited

TABLE 7. Quantitative Microscopic Findings in Lung in Inhalational Anthrax

\begin{tabular}{|c|c|c|}
\hline & Severity & $\begin{array}{l}\% \text { with } \\
\text { Feature }\end{array}$ \\
\hline Bacillus anthracis burden & $1.39 \pm 0.28$ & 49 \\
\hline$\%$ B. anthracis, intravascular & $\begin{array}{l}56.88 \pm 10.18(\text { range }=0-100 \\
\text { median }=80)\end{array}$ & \\
\hline$\%$ B. anthracis, intraalveolar & $\begin{array}{l}16.75 \pm 6.67 \text { (range }=0-95 \\
\text { median }=6)\end{array}$ & \\
\hline $\begin{array}{l}\text { Anthrax pneumonia, } \\
\text { severity }\end{array}$ & $0.515 \pm 0.113$ & 49 \\
\hline \multicolumn{3}{|l|}{ Intraalveolar exudate: } \\
\hline Transudate & $0.986 \pm 0.187$ & 60 \\
\hline Fibrin & $0.950 \pm 0.202$ & 46 \\
\hline Hemorrhage & $1.300 \pm 0.218$ & 66 \\
\hline Neutrophils & $0.297 \pm 0.096$ & 37 \\
\hline Macrophages & $1.136 \pm 0.161$ & 71 \\
\hline Lymphocytes & $0.314 \pm 0.145$ & 17 \\
\hline Plasma cells & $0.121 \pm 0.080$ & 9 \\
\hline Nuclear debris & $0.066 \pm 0.034$ & 14 \\
\hline \multicolumn{3}{|l|}{ Interstitial exudate: } \\
\hline Transudate & $1.243 \pm 0.132$ & 86 \\
\hline Fibrin & $0.772 \pm 0.142$ & 57 \\
\hline Hemorrhage & $0.943 \pm 0.153$ & 66 \\
\hline Neutrophils & $0.315 \pm 0.090$ & 43 \\
\hline Macrophages & $0.657 \pm 0.126$ & 54 \\
\hline Lymphocytes & $0.449 \pm 0.161$ & 29 \\
\hline Plasma cells & $0.300 \pm 0.125$ & 17 \\
\hline Nuclear debris & $0.057 \pm 0.033$ & 11 \\
\hline \multicolumn{3}{|l|}{ Capillaries: } \\
\hline Congestion & $2.043 \pm 0.200$ & 91 \\
\hline Capillaritis & $0.889 \pm 0.174$ & 57 \\
\hline Fibrin & $0.637 \pm 0.123$ & 75 \\
\hline Neutrophils & $1.663 \pm 0.207$ & 90 \\
\hline Macrophages & $0.763 \pm 0.223$ & 50 \\
\hline Vasculitis & $0.514 \pm 0.154$ & 66 \\
\hline Fibrin & $1.125 \pm 0.289$ & 91 \\
\hline Neutrophils & $0.875 \pm 0.223$ & 87 \\
\hline Mononuclear & $1.792 \pm 0.356$ & 91 \\
\hline \multicolumn{3}{|l|}{ Lymphangioles } \\
\hline Dilatation & $1.386 \pm 0.178$ & 74 \\
\hline Lymphangitis & $0.890 \pm 0.220$ & 37 \\
\hline Obstruction & $0.414 \pm 0.149$ & 23 \\
\hline Hemorrhage & $0.540 \pm 0.180$ & 43 \\
\hline
\end{tabular}


minimal atrophy, and phagocytosis of lymphocytes by macrophages was observed in a few cases. Red pulp: Billroth cords were markedly thickened with moderate neutrophil infiltrates and moderate-tomarked mononuclear cell infiltrates and moderate numbers of $B$. anthracis-laden macrophages. There was mild endophlebitis, minimal low-pressure hemorrhage, and rare foci of high-pressure hemorrhage. Red-pulp sinusoids were mildly to moderately congested, with mild infiltration of neutrophils and mononuclear cells. Vasculitis (fibrinoid necrosis and neutrophils) was seen in only one case and was minimal in severity.

\section{Liver}

See Table 8. Liver contained B. anthracis in 15 of 31 cases; all but one of these cases had bacilli in the sinusoids (nearly a third of the intrahepatic bacilli were in the sinusoids). All of them had bacilli in Von Kupffer cells (over half of the intrahepatic bacilli were in Von Kupffer cells). Thirteen of 15 cases had bacilli in the space of Disse, but only $10 \%$ of intrahepatic bacilli were localized there. Intrasinusoidal inflammation was present in all but two cases and consisted of mild to moderate neutrophilia, mild neutrophilic aggregates, and minimal mononuclear infiltrates. Kupffer cells were mildly to moderately hypertrophic and hyperplastic. Parenchymal cells had mild to moderate fatty metamorphosis in about half of the cases. Apoptotic hepatocytes were occasionally observed. Minimal to mild centrilobular coagulation necrosis was noted infrequently, and one case had acute viral hepatitis, and another had chronic viral hepatitis.

\section{Intestines}

The walls of the intestines (Table 9) contained $B$. anthracis in 20 of 33 patients. Grossly apprised submucosal hematomas correlated with high-pressure hemorrhages, and diffuse enteric hemorrhage correlated with low-pressure hemorrhage (Figs. 1012). Submucosal hematomas were numerous, of relatively uniform size, and small relative to those described in primary intestinal anthrax (33). Minimal vasculitis was seen in several cases and was characterized by mild-to-moderate fibrinoid necrosis and mild-to-moderate neutrophilic vasculitis. Mild-to-moderate cellulitis (diffuse neutrophil infiltration of the interstitium, usually in the submucosa) was noted in about half of the cases. In cases with early submucosal hematomas, the center of the lesion was usually in the area of the submucosa where the highest concentration of bacilli could be seen, surrounded by low-pressure hemorrhage. This hemorrhage often encroached upon the lamina propria above and the muscularis propria below to some extent but was most conspicuous in its lateral spread though the submucosa. This hemor-
TABLE 8. Quantitative Microscopic Findings in Spleen and Liver in Inhalational Anthrax

\begin{tabular}{|c|c|c|}
\hline & Severity & $\%$ with Feature \\
\hline \multicolumn{3}{|l|}{ Spleen } \\
\hline Bacillus anthracis burden & $1.664 \pm 0.307$ & 51 \\
\hline \multicolumn{3}{|l|}{ White pulp } \\
\hline \multicolumn{3}{|l|}{ Follicular } \\
\hline Atrophy & $0.471 \pm 0.089$ & 46 \\
\hline Emperiopolesis & $0.393 \pm 0.142$ & 29 \\
\hline Lymphocytolysis & $2.521 \pm 0.243$ & 89 \\
\hline \multicolumn{3}{|l|}{ Periarteriolar lymphatic sheath } \\
\hline Lymphocytolysis & $2.886 \pm 0.180$ & 100 \\
\hline Large lymphocyte hyperplasia & $2.371 \pm 0.183$ & \\
\hline \multicolumn{3}{|l|}{ Red pulp } \\
\hline \multicolumn{3}{|l|}{ Billroth cords } \\
\hline Thickening & $2.578 \pm 0.106$ & 100 \\
\hline Neutrophils & $1.793 \pm 0.138$ & 100 \\
\hline Mononuclear cells & $2.590 \pm 0.120$ & 100 \\
\hline B. anthracis-laden macrophages & $1.816 \pm 0.224$ & 83 \\
\hline Endophlebitis & $0.882 \pm 0.092$ & 83 \\
\hline Low-pressure hemorrhage & $0.338 \pm 0.109$ & 28 \\
\hline High-pressure hemorrhage & $0.029 \pm 0.029$ & 3 \\
\hline \multicolumn{3}{|l|}{ Sinusoids } \\
\hline Congestion & $1.424 \pm 0.149$ & 86 \\
\hline Neutrophils & $0.912 \pm 0.144$ & 71 \\
\hline Mononuclear cells & $1.064 \pm 0.162$ & 71 \\
\hline Vasculitis & $0.007 \pm 0.007$ & 3 \\
\hline \multicolumn{3}{|l|}{ Liver } \\
\hline B. anthracis burden & $1.258 \pm 0.269$ & 48 \\
\hline \multicolumn{3}{|l|}{$\%$ B. anthracis in } \\
\hline Sinusoids & $29.93 \pm 7.71$ & \\
\hline von Kupffer cells & $55.57 \pm 9.12$ & \\
\hline Spaces of Disse & $13.43 \pm 6.63$ & \\
\hline \multicolumn{3}{|l|}{ Intrasinusoidal inflammation } \\
\hline Neutrophils & $1.315 \pm 0.140$ & 94 \\
\hline Neutrophil aggregates & $1.177 \pm 0.174$ & 81 \\
\hline Mononuclear cells & $0.516 \pm 0.136$ & 35 \\
\hline \multicolumn{3}{|l|}{ von Kupffer cells } \\
\hline Hypertrophy & $1.605 \pm 0.178$ & 87 \\
\hline Hyperplasia & $1.326 \pm 0.153$ & 87 \\
\hline \multicolumn{3}{|l|}{ Parenchymal cells } \\
\hline Fatty metamorphosis & $0.798 \pm 0.177$ & 52 \\
\hline Apoptotic hepatocytes & $0.242 \pm 0.103$ & 23 \\
\hline Centrilobular coagulation necrosis & $0.194 \pm 0.089$ & 16 \\
\hline \multicolumn{3}{|l|}{ Viral hepatitis } \\
\hline Acute & $0.032 \pm 0.032$ & 3 \\
\hline Chronic & $0.032 \pm 0.032$ & 3 \\
\hline
\end{tabular}

rhage was surrounded by a zone of fibrin-rich edema (grossly, gelatinous edema) that varied in extent, and in turn, this was encompassed by a zone of simple edema. Mature submucosal hematomas had high-pressure hemorrhage that disrupted the central collection of bacilli.

\section{Kidneys}

See Table 9. The kidneys harbored B. anthracis in 7 of 25 cases. Glomeruli had minimal capillaritis (neutrophil clusters in capillary loops) in a few cases, and mild mesangial cell proliferation was observed occasionally, as were intratubular red blood cell casts and evidence of early acute tubular necrosis (mononuclear cells in the vasa recta).

\section{Adrenal Glands}

See Table 9. These contained B. anthracis in 7 of 16 cases. There was minimal cortical atrophy and 

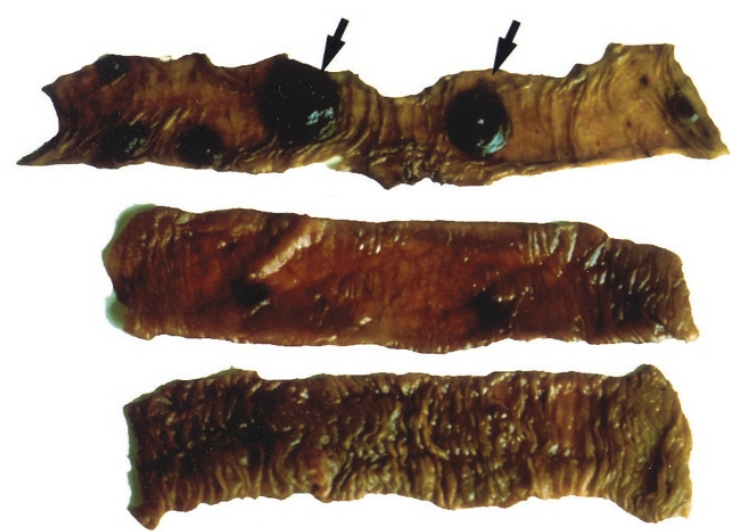

FIGURE 10. Gross photograph of intestine with diffuse enteric hemorrhage and submucosal hematomas (arrows).

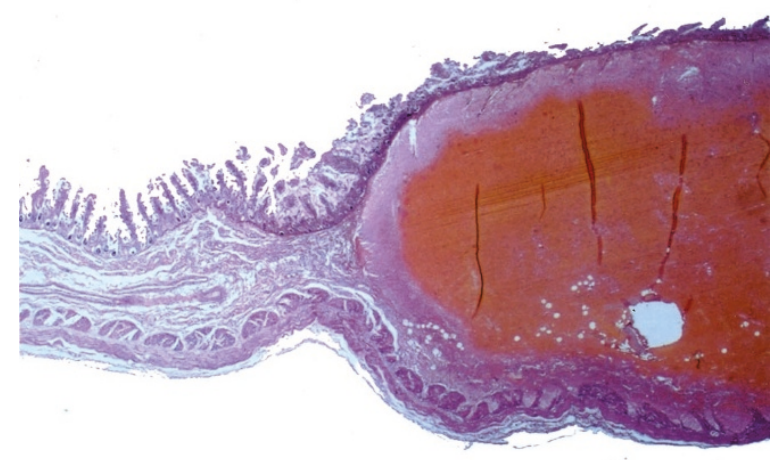

FIGURE 11. Low-magnification photomicrograph of margin of submucosal hematoma showing high-pressure hemorrhage. Hematoxylin and eosin staining, $7 \times$.

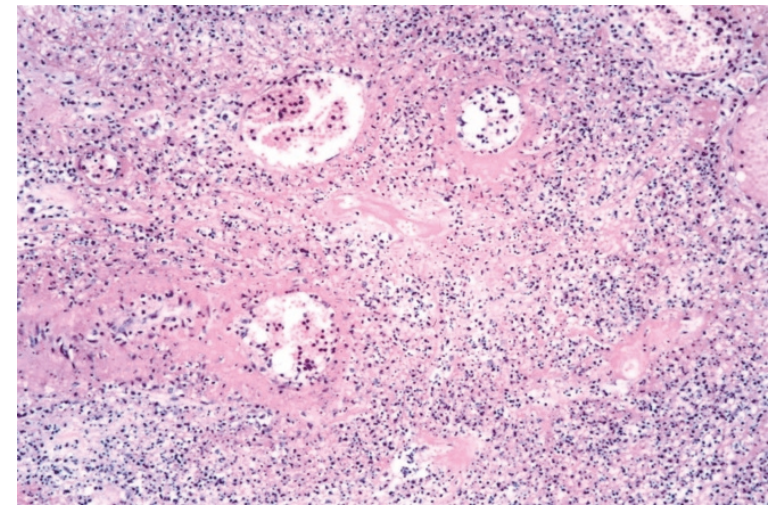

FIGURE 12. Photomicrograph of vasculitis and low-pressure hemorrhage in intestine with macroscopically diffuse enteric hemorrhage with 7-day survival. Hematoxylin and eosin staining; $100 \times$.

minimal lipid depletion. There was mild-tomoderate pseudotubular change (glandlike separation of trabeculae in the zona glomerulosa) and occasionally minimal cortical hemorrhage.

\section{Brain}

See Table 10. These samples showed microscopic lesions in the meninges and parenchyma (usually cerebral). Meninges contained B. anthracis in 23 of 29 cases (Figs. 13-16). Meninges frequently exhibited minimal-to-mild transudates, mild fibrin exudates, mild-to-moderate low-pressure hemorrhage, minimal neutrophilic and macrophage infiltrates, and less often, mild high-pressure hemorrhage. In many cases, a few lymphocytes were identified in the meninges; rare plasma cells were seen in one case, and minimal nuclear debris was noted infrequently. Moderate vasculitis was identified in several cases and was characterized by mild-tomoderate foci of fibrinoid necrosis, neutrophils, and minimal mononuclear cells. In the meninges, a similar pattern of a central focal bacillary concentration and hemorrhage surrounded by zones of fibrin-rich edema and, in turn, surrounded by simple edema was observed and appeared to have expanded progressively through the leptomeninges. In all cases in which both meninges and mediastinal lymph nodes could be examined microscopically, the stage and severity of the lesions in the mediastinum was older and more intense than in the meninges, suggesting that the mediastinal lesions preceded those in the meninges.

Cerebral parenchyma had modest mean bacillary burden. In 12 of 13 cases in which bacilli were detected in the cerebral parenchyma, the bacilli were in perivascular (Virchow-Robin) spaces. Vasculitis was composed of mild focal fibrinoid necrosis, neutrophils, and, less often, mononuclear cells. Minimal low-pressure hemorrhage was seen more frequently than high-pressure hemorrhage. Ischemic necroses, in other words, infarcts, were present in several cases, but mild diffuse acute neuronal necrosis was observed in the majority, and hypotensive sulcal necrosis was noted occasionally.

TABLE 9. Quantitative Microscopic Findings in Intestines, Kidneys, and Adrenal Glands in Inhalational Anthrax

\begin{tabular}{lcc}
\hline & Severity & \% with Feature \\
\hline Intestines & & \\
Bacillus anthracis burden & $1.879 \pm 0.350$ & 61 \\
$\quad$ Vasculitis & $0.324 \pm 0.101$ & 27 \\
$\quad$ Fibrin & $1.528 \pm 0.369$ & 89 \\
$\quad$ Neutrophils & $1.611 \pm 0.380$ & 89 \\
$\quad$ Mononuclear cells & $0.111 \pm 0.111$ & 11 \\
$\quad$ Cellulitis & $0.742 \pm 0.168$ & 52 \\
Kidneys & & \\
B. anthracis burden & $0.720 \pm 0.262$ & 28 \\
Glomerulus & & \\
$\quad$ Capillaritis & $0.390 \pm 0.147$ & 36 \\
$\quad$ Mesangial proliferation & $0.104 \pm 0.060$ & 12 \\
Tubules & & 9 \\
$\quad$ RBC casts & $0.090 \pm 0.056$ & 20 \\
$\quad$ Acute tubular necrosis & $0.273 \pm 0.188$ & \\
Adrenal Glands & & 44 \\
B. anthracis burden & $0.875 \pm 0.301$ & 81 \\
Cortical atrophy & $0.772 \pm 0.104$ & 88 \\
Lipid depletion & $0.524 \pm 0.080$ & 31 \\
Pseudotubular change & $1.625 \pm 0.268$ & \\
Hemorrhage & $0.234 \pm 0.111$ & \\
\hline
\end{tabular}


TABLE 10. Quantitative Microscopic Findings in the Central Nervous System in Inhalational Anthrax

\begin{tabular}{|c|c|c|}
\hline & Severity & $\%$ with Feature \\
\hline \multicolumn{3}{|l|}{ Brain } \\
\hline \multicolumn{3}{|l|}{ Meninges } \\
\hline Bacillus anthracis burden & $2.297 \pm 0.344$ & 79 \\
\hline Transudate & $0.759 \pm 0.152$ & 59 \\
\hline Fibrin & $0.901 \pm 0.159$ & 83 \\
\hline Low-pressure & $1.239 \pm 0.221$ & 90 \\
\hline \multicolumn{3}{|l|}{ hemorrhage } \\
\hline High-pressure & $0.603 \pm 0.240$ & 21 \\
\hline \multicolumn{3}{|l|}{ hemorrhage } \\
\hline Neutrophils & $0.524 \pm 0.142$ & 55 \\
\hline Macrophages & $0.478 \pm 0.104$ & 55 \\
\hline Lymphocytes & $0.181 \pm 0.062$ & 72 \\
\hline Plasmacytes & $0.017 \pm 0.017$ & 3 \\
\hline Nuclear debris & $0.078 \pm 0.048$ & 10 \\
\hline Vasculitis & $0.375 \pm 0.179$ & 24 \\
\hline Fibrin & $1.571 \pm 0.468$ & 71 \\
\hline Neutrophils & $1.500 \pm 0.450$ & 71 \\
\hline Mononuclear cells & $0.429 \pm 0.229$ & 43 \\
\hline \multicolumn{3}{|l|}{ Parenchyma } \\
\hline B. anthracis burden & $1.200 \pm 0.312$ & 42 \\
\hline Vasculitis & $0.342 \pm 0.107$ & 32 \\
\hline Fibrin & $1.150 \pm 0.259$ & 90 \\
\hline Neutrophils & $0.950 \pm 0.337$ & 60 \\
\hline Mononuclear cells & $0.150 \pm 0.107$ & 20 \\
\hline $\begin{array}{l}\text { Perivascular B. anthracis } \\
\text { burden }\end{array}$ & $0.683 \pm 0.203$ & 39 \\
\hline $\begin{array}{l}\text { Low-pressure } \\
\text { hemorrhage }\end{array}$ & $0.567 \pm 0.162$ & 29 \\
\hline High-pressure & $0.333 \pm 0.140$ & 19 \\
\hline hemorrhage & & \\
\hline $\begin{array}{l}\text { Ischemic necrosis } \\
\text { (infarct) }\end{array}$ & $0.227 \pm 0.091$ & 16 \\
\hline Acute neuronal necrosis & $0.705 \pm 0.072$ & 77 \\
\hline $\begin{array}{l}\text { Hypotensive sulcal } \\
\text { necrosis }\end{array}$ & $1.000 \pm 0.000$ & 10 \\
\hline
\end{tabular}

\section{DISCUSSION}

The conditions under which these autopsies were performed were comparable to those of forensic pathologists studying casualties from a mass disaster. The raw data came from the autopsy records and notes made by two of the authors (LG and FAA; clinical records were confiscated by the KGB), and museum specimens and samples preserved for microscopic examination in the autopsy suite of Hospital 40. These two factors, the work overload and the confiscation of materials, account for the incompleteness of the microscopic and gross data on each case in this set.

The rationale for this detailed study of this incomplete material was to separate the patients' other medical conditions from the "anthrax changes" by statistical analysis. Such analysis is warranted because this is the largest single autopsy series of documented human inhalational anthrax. This analysis is intended to provide the opportunity for correlation of the microscopy of human inhalational anthrax with experimental animal models. The present study emphasizes the need for more clinical and premortem laboratory information on these patients to correlate with the autopsy data, but these data remain sequestered. The opening of these data to medical scientists would very likely permit innovation of better therapies for inhalational anthrax.

The bacilli observed in histologic sections in this study are morphologically consistent with $B$. anthracis by light microscopy, and the identification was confirmed by immunohistochemical methods and culture in most of the cases. PCR done on DNA extracted from paraffin blocks from this study detected four of the five known strains of Bacillus anthracis (3). There were no quantitative bacterial cultures of the tissues and organs to corroborate the validity of the microscopic estimation of bacillary burden. Therefore, this estimate of bacterial involvement of organs is the only measured parameter of dissemination from the portal of entry or point of initial spore germination. Moreover, not all bacteria seen were strongly Gram-positive, suggesting that many may not have been viable; alternatively, the staining characteristics may have been altered by prolonged storage of the samples.

The most severe and advanced lesions in each case were in the mediastinal lymph nodes, suggesting that this involvement was the earliest in the course of the disease. This pattern is consistent with animal experiments of inhalational anthrax (15-20) in which inhaled spores are phagocytosed by alveolar macrophages and transported by these cells via pulmonary lymphangioles to the pulmonary hilar and mediastinal lymph nodes, where their germination results in dissemination of vegetative bacilli. This early involvement of mediastinal lymph nodes also indicates that these infections were acquired by inhalation, rather than ingestion, as originally reported $(1,2,27)$. The morphologic lymphangitis, spreading from the mediastinal lymph nodes, further supports a lymphohematogenous centrifugal spread from the site of initial spore germination.

\section{Epidemiological and Clinical Variables}

Patients' ages averaged in the mid-forties, with a moderate preponderance of men. The youngest man was 25 years old, and the youngest woman was the same age; thus, the age and gender demographic variables in this set of autopsied fatalities were not significantly different from that of all deaths reported or all cases, fatal or surviving (2). Surprisingly, no children or adolescents were autopsied, nor were there any children or adolescents with recorded nonfatal infections (2). It is tempting to speculate that in some manner, children are excluded from contracting or dying of inhalational anthrax. However, there are no data available on the demographic composition of the population at risk in the putative area of exposure at the time of exposure, so age- or gender-specific incidence and 
case-fatality rates cannot be determined. This area is known to be highly industrialized, and children and adolescents might indeed be underrepresented in the exposed population in factories at particular periods of the day or night. Conversely, several large apartment complexes were in the area of exposure, and children would have been expected to have been exposed there.

The mean incubation period was 16 days. The mean duration of illness before admission and duration of hospitalization (of those patients who died) was nearly 4 days and 1 day, respectively. Overall duration from date of putative exposure to death was 19.5 days; the standard errors of the means were quite wide, reflecting the great range in overall duration among the patients. Indeed, many parameters measured exhibited wide variances. It could be hypothesized that these variations result from variations in age, sex, duration, and intensity of exposure, as well as variation in the amount, timeliness, and duration of antibiotic therapy, or perhaps the heterogeneity of the strains of $B$. anthracis encountered (3). However, there is also marked variation in the incubation period in experimental animals attributed to variation in duration of the period before germination of the inhaled spores (19). The mean postmortem interval was almost 19 hours. There was wide variation, but postmortem interval had no relationship to any other measured variable in this study, so the results presented are not simply artifacts of autolysis.

The earliest tissue reaction to the presence of bacilli was edema, which apparently resulted from the adenyl cyclase action (22) of diffusing edema toxin (ET) upon adjacent endothelium; its histologic appearance is a watery expansion of the interstitial spaces around the vegetative bacilli. This was supplanted by fibrin-rich edema, in which fibrin varying in appearance from congealed amorphous masses to irregularly curvilinear interwoven strands to short straight branching and anastomosing patterns in a continuous phase of slightly eosinophilic edema was observed. The different morphologic types did not conform to any particular distribution. Fibrin-rich edema corresponds to the macroscopically described gelatinous edema, which appears as an amber gel expanding soft tissues (1). Clearly, the leakage of fibrinogen and other protein macromolecules into the interstitium represents greater endothelial permeability than simple edema. It may represent the early direct effect of lethal toxin (LT; 22) upon endothelium or the release of inflammatory mediators by macrophages triggered by LT (24). Certainly, protective antigen (PA) must diffuse more rapidly than either LF or EF because it must first bind to cells before either EF or LT can be internalized within the cell. It is probable that this fibrin-rich (gelatinous) edema corresponds to the serous inflammation of earlier Russian histopathologic descriptions (31).

That death of cells in the path of the diffusing toxins began as fibrin-rich edema was established. Both B and T lymphocytes, as well as endothelium, were highly sensitive to injury, but columnar epithelium and glandular cells of varying types appeared relatively intact. Lymphocyte death morphologically resembled apoptosis and resulted in faintly basophilic debris. It involved both B-cell and T-cell zones of lymph nodes and spleen similarly but was also prominent near clusters of bacilli. Lethal toxin interferes with intracellular signaling (23) and may induce apoptosis. Inflammatory response was scant compared with the magnitude of necrosis/apoptosis, but neutrophils were prominent in patients with shorter survival, whereas mononuclear cells (large lymphocytes, immunoblasts, and macrophages) were significantly associated with longer survival. Previous reports of both human (11-15) and experimental (16-21) inhalational and intestinal (31) anthrax variably report neutrophilic infiltrates associated with the hemorrhagic, necrotic tissue injury in anthrax; most descriptions include some, even significant, neutrophil infiltrates. Whereas the average magnitude of the neutrophil exudate in this series was low in most affected tissues, it was prominent in some locations in some cases and prominent in some organs, and not others, in other cases. We conclude that a neutrophilic infiltrate is generally present but is scant in comparison to the amount of cell death. This would correlate with an apoptotic mechanism of cell death. Most of the lesions implicate toxin injury and meager neutrophilic response; patients surviving longer appear to shift from neutrophils to mononuclear leukocytes, especially in the vasculitis (vide infra).

Vasculitis consisted of endothelial adherence by inflammatory cells, endothelial necrosis with intravascular fibrin deposition, inflammatory cell infiltrate into the vascular intima and media, and fibrinoid necrosis of vessel walls. Frequency and severity of vasculitis in all sites were averaged to give an overall estimate of the phenomenon in each case. Vasculitis, including capillaritis, was prominent in all cases. Vasculitis, excluding capillaritis, was moderate to marked. Analysis of the vasculitis in various organs (Tables 4-10) revealed that vascular fibrinoid necrosis was seen with equal frequency and severity in shorter and longer durations of illness, whereas neutrophils were predominant in patients with shorter survival, and mononuclear cells dominated in longer survivors. The pathogenic mechanism of this vasculitis is not known. It may be a direct or indirect effect of the toxins produced by the bacilli and could be related to release of proinflammatory cytokines (24). Immunohistochemical 


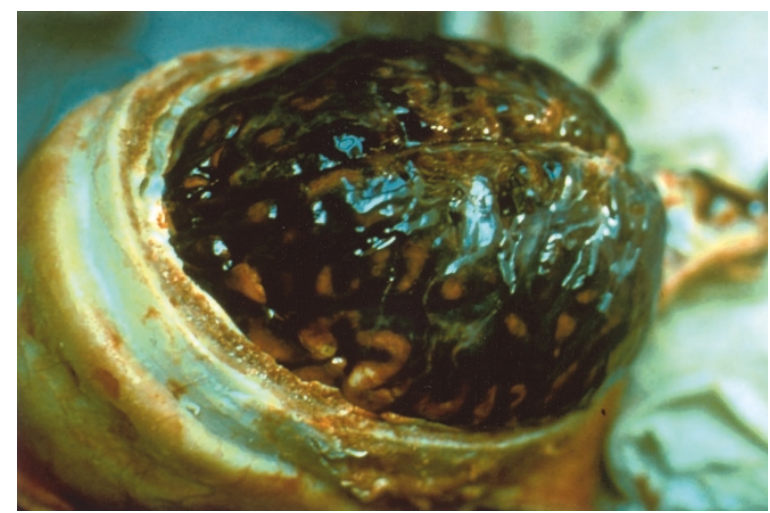

FIGURE 13. Gross photograph of hemorrhagic meningitis in situ in a case of fatal inhalational anthrax.

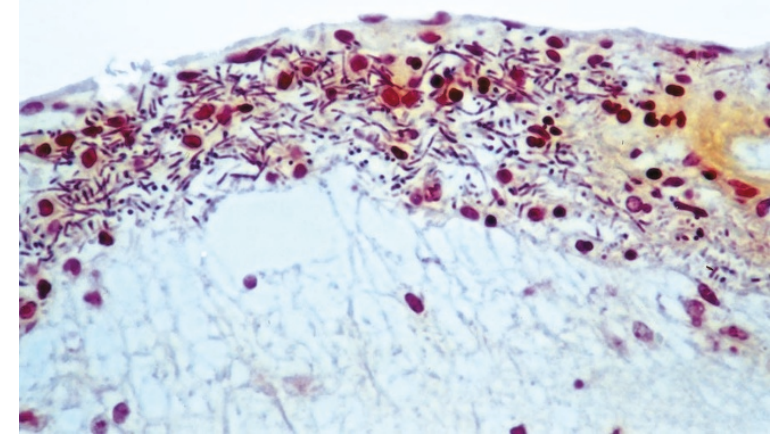

FIGURE 14. Photomicrograph of $B$. anthracis in the subarachnoid space of the meninges. Brown-Hopps stain; $200 \times$.

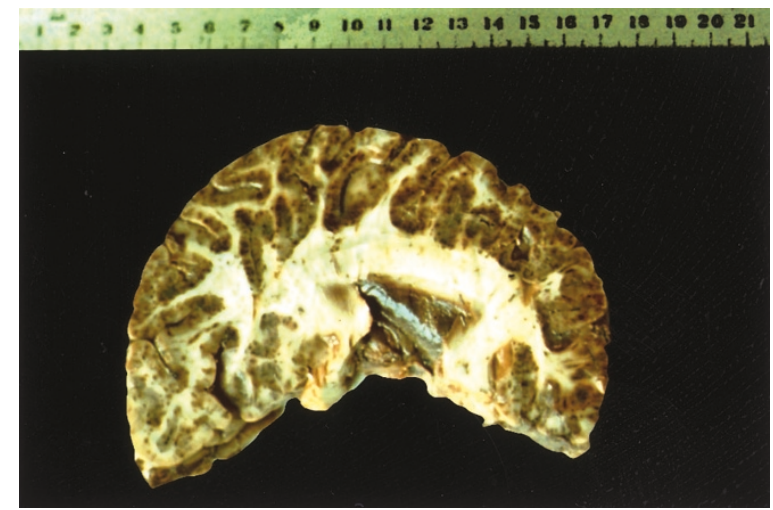

FIGURE 15. Gross photograph of hemorrhagic encephalitis in case of fatal inhalational anthrax.

study revealed some IgM and/or IgG, but no complement, within some vessel walls; therefore, the distribution of these proteins may be a result of immunoglobulin leakage into damaged vessels, rather than causing the vasculitis. Vasculitis has not been reported in previous studies of human inhalational anthrax (11-15), nor was it identified in experimental studies (16-21), but vasculitis and even microaneurysms were recognized by Russian workers, as significant constituents in the pathogenesis of intestinal anthrax; vascular injury was even postulated to be responsible for some of the

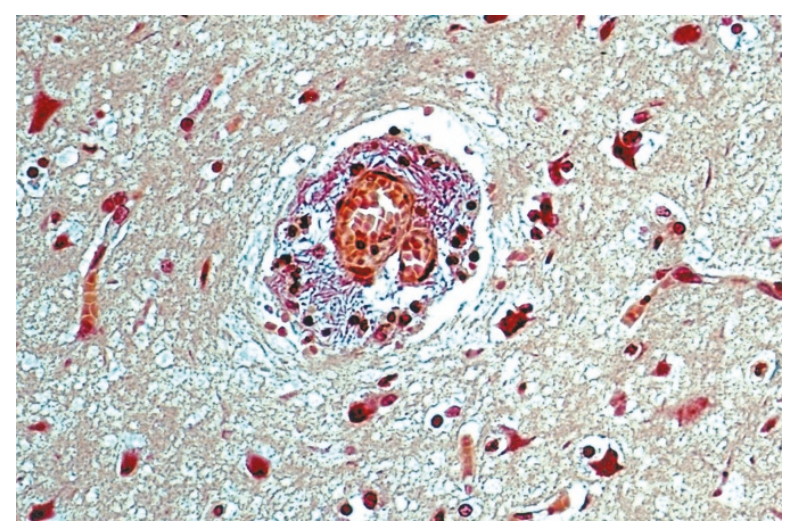

FIGURE 16. Photomicrograph of B. anthracis filling Virchow-Robin space in cerebral cortex. Brown-Hopps staining; $200 \times$.

hemorrhage observed (31). Derizhanov's (31) histopathologic observations of the lesions in autopsies of patients dying of intestinal anthrax were remarkably coincident with our observations, except that the primary site was distal small intestine and cecum with initial spread to mesenteric lymph nodes rather than the lungs and mediastinal lymph nodes, respectively, in these inhalational-anthrax cases.

High pressure hemorrhage, hemorrhage which compressed and displaced the adjacent tissues, was prominent in all cases. Although these hemorrhages probably result from the vascular rupture on the arterial side of the microcirculation, perhaps due to infection-induced aneurysms, as speculated by Derizhanov (31), demonstrating such small focal phenomena is more difficult than finding the larger hemorrhages, and the hemorrhage would very likely obliterate the small vascular lesion of origin. Such hemorrhages would be expected to occur in loci with more dense concentrations of bacilli, where high toxin concentrations and tissue damage would be maximal.

Low-pressure hemorrhages, those in which erythrocytes permeate interstitial tissues, clearly result from increased endothelial permeability and/or necrosis, probably on the venular side of the microcirculation. The injury involves vascular basement membranes and mural components of vessels with relatively low intraluminal pressure; it is most probable that this damage results from bacillary toxin injury to the smallest, thinnest-walled vessels, the capillaries and venules.

Hemorrhagic diatheses were assessed macroscopically by the presence of multifocal petechiae and ecchymoses in the skin, serosal surfaces, and visceral parenchyma and were present in 29 of the 40 cases in which data were available. Although hemorrhage was probably in part a result of the toxic vascular injury, one of the authors (OY), who was active in treatment of these patients and others 
who survived, believes that the hemorrhagic diatheses seen at autopsy were, in part, the result of disseminated intravascular coagulation. She recollects that the patients generally had decreased fibrinogen, increased bleeding time, marked prolongation of clotting time, and decreased platelets.

The apparent clinical well-being of patients with systemic anthrax during most of the course of the disease and then the sudden onset of septic shock has been a consistent observation in both experimental and human anthrax (2, 9-15, 19-21, 31). The usual stigmata of septic hypotension and splanchnic vasoconstriction (centrilobular necrosis of the liver, acute tubular necrosis of the kidneys, ARDS, superficial hemorrhagic necrosis of the intestines and cerebral watershed injuries) were encountered rather infrequently among these cases. This suggests that septic hypotension, which was seen clinically, was so precipitous and profound that its sequelae had no time to develop. Indeed, short hospital courses were the rule in these fatal cases.

These human data are consistent with a portal of entry in the lower respiratory tract, with phagocytosis and transport of spores by macrophages to pulmonary hilar and mediastinal lymph nodes, where germination and vegetative bacillary proliferation ensued. Subsequent transport of proliferating bacilli via the lymphatics would be enhanced by increased lymph flow and would disseminate the bacilli through the thoracic duct into the blood, as well as via retrograde lymphangitic spread back into the perihilar regions of the lungs. Hematogenous spread to all other organs followed. In each disseminated site, bacilli proliferated first within vessels and then extravascularly and spread in concentrically enlarging spheres through the interstitium and within draining lymphatic channels. This spread appeared to be facilitated by the edema that accompanied bacillary proliferation. These findings corroborate the validity of experimental models (16-21) for the human disease.

The composition of the lesions in the mediastinal lymph nodes and soft tissues, as well as in "metastatic" sites was similar. Initial bacillary proliferation occurs, followed by transudation of edema, implying a loss of endothelial integrity; further endothelial compromise results in leakage of fibrinogen and its polymerization to form the fibrin-rich or "gelatinous" edema, an amber gelatinous expansion of the soft tissue central to the more fluid edema. Finally, cellular death, probably apoptotic rather than necrotic, judging by the sparse neutrophilic response, ensues with loss of vascular integrity and either low-pressure or high-pressure hemorrhage. In low-pressure hemorrhage, erythrocytes infiltrate parenchyma (often already necrotic) in a diffuse manner without displacing tissue architecture, whereas in high-pressure hemorrhage, these reticular architectures are clearly displaced by large lakes of erythrocytes. We hypothesize that low-pressure hemorrhages occur by compromise of endothelium of low-pressure vessels, such as capillaries and venules, whereas high-pressure hemorrhages resulted from rupture of high-pressure vessels, such as small arteries and arterioles.

The composition of lesions observed in these inhalational anthrax cases (peripheral simple edema surrounding a zone of gelatinous edema, in turn encompassing a central zone of necrosis and hemorrhage) resembles other forms of anthrax, such as cutaneous anthrax, where a desiccated central hemorrhagic necrotic lesion is surrounded by rings of gelatinous edema and then simple edema. This pattern corresponds with the pathophysiologic hypothesis of differential diffusion of three proteins: first PA, then EF and LF, from foci of bacillary proliferation. In such a model, EF diffuses more rapidly than LF. LF interrupts intracellular communication, resulting in cell death, but also (at lower concentrations) causes release of inflammatory mediators (IL-1 and TNF- $\alpha$ ), which may produce the profound septic hypotension seen terminally.

We have relearned and refined the basic histopathology and pathogenesis of anthrax, initially discerned from observations on humans $(31,32)$ and in experimentally infected animals (11-15). This large series confirms previous reports of the pathologic changes in human inhalational anthrax (1621) but reveals the greater range of variation in the disease. This information would be particularly important to attending pathologists if and when the first cases of deliberate bioterrorist dispersal of anthrax come to pass.

Acknowledgments: The authors thank Kelly Cassity for expert secretarial assistance in the preparation of the manuscript and the advice of Dr. Benjamin Gelman in interpretation of the central nervous system microscopic lesions.

\section{REFERENCES}

1. Abramova FA, Grinberg LM, Yampolskaya OV, Walker DH. Pathology of inhalational anthrax in 42 cases from the Sverdlovsk outbreak of 1979. Proc Natl Acad Sci U S A 1993;90: 2291-4.

2. Meselson M, Guillemin J, Hugh-Jones M, Langmuir A, Popova I, Shelokov A, et al. The Sverdlovsk anthrax outbreak of 1979. Science 1994;266:1202-08.

3. Jackson PJ, Hugh-Jones ME, Adair DM, Green G, Hill KK, Kuske CR, et al. PCR analysis of tissue samples from 1979 Sverdlovsk anthrax victims: the presence of multiple Bacillus anthracis strains in different victims. Proc Natl Acad Sci U S A 1997;95:1224-29.

4. Meselson M. The biologic weapons convention and the Sverdlovsk anthrax outbreak of 1979. J Fed Am Scientists 1988; 41:1-6.

5. Bezdenzhnykh IS, Nikiforov VN. Epidemiologic analysis of anthrax in Sverdlovsk. Zh Mikrobiol Epidemiol Immunobiol 1980;5:111-13. 
6. Gumbel P. The anthrax mystery. Wall Street J, October 21, 1991.

7. Gumbel P. Death in the air. Wall Street J, October 22, 1991.

8. Gumbel P. Anthrax: The survivors speak. Wall Street J, October 23, 1991.

9. Albrink WS, Brooks SM, Biron RE, Kopel M. Human inhalation anthrax: A report of three fatal cases. Am J Pathol 1960; 61:457-71.

10. Brachman PS, Pagano JS, Albrink WS. Two cases of fatal pulmonary anthrax, one associated with sarcoidosis. N Engl J Med 1961;265:203-8.

11. Entiknap JB, Galbratih NS, Tomlinson AJH, Elias-Jones TF. Pulmonary anthrax caused by contaminated sacks. Br J Ind Med 1968;25:72-4.

12. La Force FM, Bumford FH, Feeley JC, Stokes SL, Snow DB. Epidemiologic study of a fatal case of inhalation anthrax. Arch Environ Health 1969;18:798-805.

13. Vessal K, Yeganehdoust J, Dutz W, Kohout E. Radiologic changes in inhalation anthrax. A report of radiological and pathological correlation in two cases. Clin Radiol 1975;26: 471-4.

14. Severn M. A fatal case of pulmonary anthrax. Br Med J 1976;1:748.

15. Suffin SC, Carnes WH, Kaufman AF. Inhalation anthrax in a home craftsman. Hum Pathol 1978;9:594-7.

16. Young GA, Zelle MR, Lincoln RE. Respiratory pathogenicity of Bacillus anthracis spores. I. Methods of study and observation on pathogenesis. J Infect Dis 1946;79:233-46.

17. Barnes JM. The development of anthrax following administration of spores by inhalation. Br J Exp Pathol 1947;28:38594.

18. Ross JM. The pathogenesis of anthrax following the administration of spores by the respiratory route. J Pathol Bacteriol 1957;73:485-94.

19. Henderson DW, Peacock S, Belton FC. Observations on the prophylaxis of experimental pulmonary anthrax in the monkey. J Hyg 1956;54:28-36.

20. Albrink WS, Goodlow RJ. Experimental inhalation anthrax in the chimpanzee. Am J Pathol 1959;35:1055-65.
21. Albrink WS. Pathogenesis of inhalation anthrax. Bacteriol Rev 1961;25:268-73.

22. Aroura N, Leppla SH. Residues 1-254 of anthrax toxin lethal factor are sufficient to cause cellular uptake of fused polypeptides. J Biol Chem 1993;268:3334-41.

23. Duesbery NS, Webb CP, Leppla SH, Gordon VM, Klimpel KR, Copeland TD, et al. Proteolytic inactivation of MAP kinase-kinase by anthrax lethal factor. Science 1998;280:734-7.

24. Hanna PC, Acosta D, Collier RJ. On, the role of macrophages in anthrax. PNAS 1993;90:10198-201.

25. Abramova AA, Grinberg LM. [Pathology of anthrax sepsis according to materials of the infectious outbreak in 1979 in Sverdlovsk (macroscopic changes).] Arkh Patol 1993;55:1217.

26. Abramova AA, Grinberg LM. [Pathology of anthrax sepsis according to materials of the infectious outbreak in 1979 in Sverdlovsk (microscopic changes).] Arkh Patol 1993;55:1823.

27. Grinberg LM, Abramova AA. [Pathology of anthrax sepsis according to materials of the infectious outbreak in 1979 in Sverdlovsk (various aspects of morpho-, patho- and thanatogenesis).] Arkh Patol 1993;55:23-6.

28. Grinberg LM, Abramova FA, Yampolskaya OV, Smith JH, Walker DH. Pathology of human inhalation anthrax [Abstract]. Mod Pathol 1998;11:144A.

29. Grinberg LM, Abramova FA, Yampolskaya OV, Smith JH, Walker DH. Quantitative microscopic findings in inhalational anthrax [Abstract]. In: Program and abstracts of the $46^{\text {th }}$ Annual Meeting of the American Society of Tropical Medicine and Hygiene 1997;57:128.

30. Grinberg LM, Abramova FA, Yampolskaya OV, Smith $\mathrm{JH}$, Walker DH. Quantitative microscopic findings in inhalational anthrax [Abstract] Am J Clin Pathol 1998;109: 480A.

31. Derizhanov SM, Pathologic anatomy and pathogenesis of the intestinal form of anthrax. Smolensk, Russia: Western Oblast Governmental Printing House; 1935.

32. Dutz W, Kahout E. Anthrax. Pathol Annu 1971;6:209-48. 